NT@UW-98-20

\title{
Rotational Invariance In Nuclear Light-Front Mean Field Theory
}

\author{
P. G. Blunden \\ Department of Physics and Astronomy \\ University of Manitoba \\ Winnipeg, MB, Canada R3T 2N2 \\ M. Burkardt \\ Department of Physics \\ New Mexico State University \\ Las Cruces, NM 88003-0001, U.S.A. \\ G. A. Miller \\ Department of Physics, Box 351560 \\ University of Washington \\ Seattle, WA 98195-1560, U.S.A.
}

(April 16, 2018)

\begin{abstract}
A light-front treatment for spherical nuclei is developed from a relativistic effective Lagrangian and employing the mean field approximation. Minimizing the nuclear minus momentum subject to the constraint that, in the rest frame, the expectation values of the plus- and minus-momenta are the same leads to a formalism in which rotational invariance is recovered.
\end{abstract}

Light cone variables have long been used to analyze high energy experiments involving 
nuclear and nucleon targets. The most well-known use appears in the parton model. The Bjorken variable $x$ is the ratio $x=k^{+} / p^{+}$where $k^{+}=k^{0}+k^{3}$ is the plus-momentum of the struck quark and $p^{+}$is the plus-momentum of the target. Quark distributions represent the probability that a quark has a plus-momentum fraction $x$. Our focus here is on the distribution functions which describe the plus-momentum carried by the nucleons within the nucleus. Such distributions, which depend on $p^{+}$of the struck nucleon, are needed to analyze deep inelastic lepton-nucleus scattering [1], and also enter in the analysis of highmomentum transfer nuclear quasi-elastic reactions such as $\left(e, e^{\prime}\right),\left(e, e^{\prime} p\right),(p, 2 p)$ [2]- [4]. One may also obtain similar distributions for the nuclear mesons, and these enter in the analyses of nuclear Drell-Yan experiments [5]. If the light front formalism is used, these distributions are simply related to the square of the nuclear ground state wave functions [6]. Many nuclear high momentum transfer experiments are planned, so that it is necessary to derive a relativistic formulation which uses $p^{+}$variables closely related to experiments, and which incorporates the full knowledge of nuclear dynamics.

If the relevant nuclear wave functions depend on $p^{+}$, the canonical spatial variable is $x^{-}=x^{0}-x^{3}$. This leaves $x^{+}=x^{0}+x^{3}$ to be used as a time variable, with the light front Hamiltonian $\left(x^{+}\right.$development operator) as $P^{-}=P^{0}-P^{3}$. These are the light front variables of Dirac [7]. There are clear advantages in using these variables, but a principal problem arises because the use of $x^{+}$as "time" and $x^{-}, \boldsymbol{x}_{\perp}$ as "space" involves the loss of manifest rotational invariance. This is especially important in nuclear physics because the understanding of magic numbers rests on the $2 j+1$ degeneracy of single particle orbitals of good angular momentum. Our purpose is to show how rotational invariance is recovered in nuclear mean field theory. Once this is accomplished, light front dynamics can be a useful tool in a variety of nuclear physics calculations.

We start by assuming a field-theoretic Lagrangian in which the nuclear constituents are nucleons $\psi$ scalar mesons $\phi$ and vector mesons $V^{\mu}, V^{\mu \nu}=\partial^{\mu} V^{\nu}-\partial^{\nu} V^{\mu}$. The Lagrangian is a standard one [8], and we use the meson-nucleon coupling constants and meson masses of Horowitz and Serot 9]. The use of light front dynamics allows us to compute the necessary 
nuclear meson distribution functions using the same formalism that yields the nuclear binding energy and density. Previously, one of us constructed a light front mean-field treatment of nuclear matter [10] using the same Lagrangian. Vector mesons were found to carry $35 \%$ of the total plus-momentum (mass), with each vector meson carrying an infinitesimally small amount of plus-momentum. Does such a result hold for finite nuclei?

Light front quantization of a given Lagrangian is a standard procedure [6]. One first uses the Lagrangian to derive field equations which enable the identification of dependent degrees and their elimination in favor of independent ones. The procedure for the present Lagrangian is described elsewhere [10], so we discuss only the most relevant features concerning the nucleons. Two of the four degrees of freedom of this spin $1 / 2$ particle entering in the usual Dirac spinor must be redundant. These are separated using the projection operators $\Lambda_{ \pm} \equiv \frac{1}{2} \gamma^{0} \gamma^{ \pm}$, with $\psi_{ \pm} \equiv \Lambda_{ \pm} \psi$. The independent fermion degree of freedom is chosen as $\psi_{+}$. In general the equation for $\psi_{-}$depends on the interaction $V^{+}$in a complicated manner. This dependence is eliminated by using the transformation of Refs. [11]. The net result is that a transformed vector meson field $\bar{V}^{\mu}=V^{\mu}-\frac{\partial^{\mu}}{\partial^{+}} V^{+}$enters in the nucleonic field equation, while $V^{\mu}$ enters in the equation for the vector meson field.

The energy momentum tensor is $T^{\mu \nu}$, and the volume integrals of components $T^{+-}$and $T^{++}$are the Hamiltonian $P^{-}$and momentum $P^{+}$. We find

$$
\begin{aligned}
& T^{+-}=\left(\boldsymbol{\nabla}_{\perp} \phi\right) \cdot\left(\boldsymbol{\nabla}_{\perp} \phi\right)+m_{s}^{2} \phi^{2}-\left(\boldsymbol{\nabla}_{\perp} V^{+}\right) \cdot\left(\boldsymbol{\nabla}_{\perp} V^{-}\right)-m_{v}^{2} V^{+} V^{-}+2 \psi_{+}^{\dagger}\left(i \partial^{-}-g_{v} \bar{V}^{-}\right) \psi_{+}, \\
& T^{++}=\partial^{+} \phi \partial^{+} \phi+\left(\boldsymbol{\nabla}_{\perp} V^{+}\right) \cdot\left(\boldsymbol{\nabla}_{\perp} V^{+}\right)+m_{v}^{2} V^{+} V^{+}+2 \psi_{+}^{\dagger} i \partial^{+} \psi_{+} .
\end{aligned}
$$

We choose to work in the nuclear rest frame so that $P_{\perp}|\Psi\rangle=0$ and $P^{+}|\Psi\rangle=M_{A}|\Psi\rangle$. In this frame, the light-front Schrödinger equation for the ground-state wave function $|\Psi\rangle$ reads

$$
P^{-}|\Psi\rangle=\frac{M_{A}^{2}+P_{\perp}^{2}}{P^{+}}|\Psi\rangle=M_{A}|\Psi\rangle
$$

In light-front dynamics the goal is to solve the equation $\left(P^{+} P^{-}-P_{\perp}^{2}\right)|\Psi\rangle=M_{A}^{2}|\Psi\rangle$ with fixed values for $P_{\perp}$ and $P^{+}$, using a Fock-space basis. This is not possible for systems 
containing many nucleons, so we need to construct a light front version of the HartreeFock procedure in which the energy of a Slater determinant is minimized. Minimizing the expectation value of $P^{-}$doesn't work because one gets a zero of $P^{-}$for an infinite value of $P^{+}$. Instead we minimize the expectation value of $P^{-}$subject to the condition that the expectation value of $P^{+}$is equal to the expectation value of $P^{-}$. This is the same as minimizing the average of $P^{-}$and $P^{+}$. To this end we define a light front Hamiltonian: $H_{L F} \equiv \frac{1}{2}\left(P^{+}+P^{-}\right)$. This is not the usual Hamiltonian because light-front quantization is used to define all of the operators that enter.

The wave function $|\Psi\rangle$ consists of a Slater determinant of nucleon fields $|\Phi\rangle$ times a mesonic portion, and the mean field approximation is characterized by the replacements: $\phi \rightarrow\langle\Psi|\phi| \Psi\rangle, V^{\mu} \rightarrow\left\langle\Psi\left|V^{\mu}\right| \Psi\right\rangle$. We quantize the nucleon fields using

$$
\psi(x)=\sum_{n}\left\langle x^{-}, x_{\perp} \mid n\right\rangle e^{-i p_{n}^{-} x^{+} / 2} b_{n}
$$

in which the variable $x$ represents both the spatial variables $\boldsymbol{x}_{\perp}, x^{-}$and the spin, isospin indices, and $b_{n}$ obeys the usual anti-commutation relations. The meson fields are also functions of $x^{-}, x_{\perp}$. The single particle states $|n\rangle$ are defined by minimizing the nucleonic contribution to the expectation value of $\left\langle\Psi\left|H_{L F}\right| \Psi\right\rangle$. The Slater determinant $|\Phi\rangle$ is defined by allowing $A$ nucleon states to be occupied. The results of the minimization are

$$
\begin{aligned}
p_{n}^{-}|n\rangle_{+} & =\left(i \partial^{+}+2 g_{v} \bar{V}^{-}\right)|n\rangle_{+}+\left(\boldsymbol{\alpha}_{\perp} \cdot\left(\boldsymbol{p}_{\perp}-g_{v} \overline{\boldsymbol{V}}_{\perp}\right)+\beta\left(M+g_{s} \phi\right)\right)|n\rangle_{-}, \\
i \partial^{+}|n\rangle_{-} & =\left(\boldsymbol{\alpha}_{\perp} \cdot\left(\boldsymbol{p}_{\perp}-g_{v} \overline{\boldsymbol{V}}_{\perp}\right)+\beta\left(M+g_{s} \phi\right)\right)|n\rangle_{+},
\end{aligned}
$$

where $|n\rangle_{ \pm}=\Lambda_{ \pm}|n\rangle$. Minimization of $\left\langle\Psi\left|H_{L F}\right| \Psi\right\rangle$ with respect to the meson field variables leads to the meson field equations. We find

$$
\begin{gathered}
\left(-\nabla_{\perp}^{2}-\left(2 \frac{\partial}{\partial x^{-}}\right)^{2}+m_{s}^{2}\right)\langle\Psi|\phi(x)| \Psi\rangle=-g_{s} \rho_{s}(x), \\
\left(-\nabla_{\perp}^{2}-\left(2 \frac{\partial}{\partial x^{-}}\right)^{2}+m_{s}^{2}\right)\left\langle\Psi\left|V^{\mu}(\boldsymbol{r})\right| \Psi\right\rangle=g_{v} j_{B}^{\mu}(x),
\end{gathered}
$$

where $\rho_{s}(x) \equiv\langle\Psi|\bar{\psi}(x) \psi(x)| \Psi\rangle$ and $j_{B}^{\mu}(x) \equiv\left\langle\Psi\left|\bar{\psi}(x) \gamma^{\mu} \psi(x)\right| \Psi\right\rangle$. 
We next discuss how Eq. (4) is solved. The first step is to use a representation 12 in which $|n\rangle_{ \pm}$are each represented as Pauli spinors. The light front quantization respects manifest rotational invariance for rotations about the $z$-axis. Thus each single-particle state has a good $J_{z}$. We use a momentum representation for the longitudinal variable in which the values of $p^{+}$take on those of a discrete set: $p_{m}^{+}=(2 m+1) \pi /(2 L)$ where $m \geq 0$ and $L$ is a quantization length. This means that the nucleon wave functions have support only for $p^{+} \geq 0$. This spectrum condition is a requirement for exact solutions for any theory. The coordinate space representation is used for the $\perp$ variables. Then we have

$$
\left\langle p_{m}^{+}, \boldsymbol{x}_{\perp} \mid n\right\rangle_{+}=\left[\begin{array}{c}
U_{m}^{(n)}\left(x_{\perp}\right) e^{i\left(J_{z}-1 / 2\right) \phi} \\
L_{m}^{(n)}\left(x_{\perp}\right) e^{i\left(J_{z}+1 / 2\right) \phi}
\end{array}\right],
$$

in which the upper (lower) entrees of the Pauli spinor correspond to $m_{s}=1 / 2(-1 / 2)$, and $\boldsymbol{x}_{\perp} \equiv\left(x_{\perp}, \phi\right)$. When Eq. (7) is used in Eq. (四), one finds that the equations do not depend on the magnitude of $J_{z}$; solutions for \pm a given magnitude of $J_{z}$ are degenerate. The functions $U_{m}\left(x_{\perp}\right)$ and $L_{m}\left(x_{\perp}\right)$ are expanded in a basis of B-splines of degree five in $x_{\perp}$ [13. One starts with a guess for the scalar and vector potentials. Then one solves Eq. (4). The solutions are used to generate new scalar and vector nucleon densities, which in turn generate new potentials via Eqs.(5) and (6). This iteration procedure is completed until the solutions are stable.

If these solutions are to have any relevance at all, they should respect rotational invariance. The success in achieving this is examined in Table I, which gives our results for the spectrum of ${ }^{40} \mathrm{Ca}$. We see that the $J_{z}= \pm 1 / 2$ spectrum contains the eigenvalues of all states, since all states must have a $J_{z}= \pm 1 / 2$ component. Furthermore, the essential feature that the expected degeneracies among states with different values of $J_{z}$ are reproduced numerically.

The results shown in Table 1 are obtained using a basis of 20 splines, a box size of $2 L=24 \mathrm{fm}$, and 24 Fourier components in the expansion of the wavefunction. This value of $L$ is large enough so that our results do not depend on it, and the number of terms in the expression for the density is enough to ensure that the densities are spherically symmetric 
[14]. Another feature is that the spectrum with $p^{+}>0$ has no negative energy states, so that in using the LF method one is working in a basis of positive energy states only.

The single particle energies $\epsilon_{n}$ reported in Table 1 are given by $\epsilon_{n}=p_{n}^{-} / 2$. The values of $\epsilon_{n}$ are essentially the same as those of the ET formalism to within the expected numerical accuracy of our program, as are the nuclear densities. This equality is not mandated by spherical symmetry alone because the solutions in the equal time framework have nonvanishing components with negative values of $p^{+}$. We may understand the near equality of single particle eigenvalues using an analytic argument. First we use a representation in which $V^{\mu}$ appears in the equation for the nucleon field. That is, define $\langle x \mid n\rangle^{\prime} \equiv e^{-i g_{v} \Lambda(x)}\langle x \mid n\rangle$, with $\partial^{+} \Lambda=V^{0}$. Then multiply the equation for $|n\rangle_{+}^{\prime}$ by $\gamma^{+}$and the equation for $|n\rangle_{-}^{\prime}$ by $\gamma^{-}$. Adding the resulting two equations gives

$$
\left(\gamma^{0}\left(p_{n}^{-}-2 \gamma^{0} g_{v} V^{0}-\gamma^{3}\left(2 p^{+}-p_{n}^{-} / 2\right)\right)|n\rangle^{\prime}=2\left(\gamma_{\perp} \cdot \boldsymbol{p}_{\perp}+M+g_{s} \phi\right)|n\rangle^{\prime} .\right.
$$

Solving Eq. (8) in coordinate space is expected to lead to solutions in which the spectrum condition is not respected exactly. Equation (8) may be converted into a manifestly rotationally invariant equation using the relation: $x^{-}=-2 z$, so that $p^{+}=i \partial^{+}=2 i \frac{\partial}{\partial x^{-}} \rightarrow-i \frac{\partial}{\partial z}$. The operator $p^{+}$acts as a $p^{3}$ operator, and the result (8) looks like the Dirac equation of the equal time (ET) formulation (of eigenvalue $p_{n}^{-} / 2$ ) except for an offending term $-p_{n}^{-} / 2$ multiplying the $\gamma^{3}$. This term may be eliminated by including a phase factor: $\langle x \mid n\rangle^{\prime}=e^{i p_{n}^{-} z / 2}\langle x \mid n\rangle_{E T}$. The result is that $\langle x \mid n\rangle_{E T}$ satisfies the standard Dirac equation of the equal time formulation. This means that the eigenvalues $\epsilon_{n}$ must be approximately the same in the two formulations. The net result of these transformations is that if one were to include $p^{+}<0$ in the LF calculation then the eigenvalues $\varepsilon_{n}$ in the LF and ET calculations would be identical and the LF wave function would be expressed as

$$
\left\langle p^{+}, x_{\perp} \mid n\right\rangle=\frac{1}{\sqrt{2 \pi}} \int_{-\infty}^{\infty} d z e^{-i\left(p^{+}-p_{n}^{-} / 2\right) z} e^{i g_{v} \Lambda\left(x_{\perp}, z\right)}\left\langle z, x_{\perp} \mid n\right\rangle_{E T} .
$$

The relation (9) tells us that $\left\langle p^{+}, x_{\perp} \mid n\right\rangle$ peaks at $p^{+} \approx M-g_{V} V^{0}$, with a width of the order of the inverse of the radius of the entire nucleus. Therefore, neglecting $p^{+}<0$ is only 
a minor approximation, which is the reason why our above LF calculation, with $p^{+}<0$ suppressed, leads to eigenvalues $\varepsilon_{n}$ that are approximately equal to the ET results.

One can also read off from Eq. (9) that the influence of the vector potential is to remove plus-momentum from the nucleons. Furthermore, the large value of the nuclear radius causes the region of support to be very narrow, so that $\left\langle p^{+}, x_{\perp} \mid n\right\rangle$ is very small for negative values of $p^{+}$.

The nuclear energy is obtained by adding the contributions to the expectation value of $P^{-}+P^{+}$from the nucleons, and scalar and vector mesons. Then straightforward calculation leads to the result

$$
M_{A}=\sum_{n<F} \epsilon_{n}-\frac{1}{2} \int d^{3} r\left[g_{s} \phi(r) \rho_{s}(r)+g_{v} V^{0}(r) \rho_{B}(r)\right],
$$

where $\boldsymbol{r} \equiv\left(\boldsymbol{x}_{\perp}, z=-x^{-} / 2\right)$. The above expression is the result obtained in standard equal time calculations, and the nuclear binding energy we obtain is essentially the same as that of Ref. [9].

The previous results mean that we have reproduced the standard good results for nuclear binding energy and density using a formalism that allows us to compute nucleon and meson plus-momentum distributions used to analyze various high energy nuclear experiments. The probability that a nucleon $f_{N}\left(p^{+}\right)$has a momentum $p^{+}$is given simply by

$$
f_{N}\left(p^{+}\right)=\sum_{n<F} \int d^{2} x_{\perp}\left|\left\langle\mathbf{x}_{\perp}, p^{+} \mid n\right\rangle\right|^{2} .
$$

It is convenient to express the distribution in terms of the dimensionless variable $y \equiv$ $p^{+} /\left(M_{A} / A\right)$. The result is shown in Fig. 1 . The peak of the distribution is at $y \approx 0.76$, corresponding to $p^{+}$of $710 \mathrm{MeV}$ (which is the plus-momentum per nucleon). Our numerical calculations show that the average value of $p^{+}$is $\left.672.56 \mathrm{MeV}(<y\rangle=0.72\right)$ out of a total expectation value $P^{+} / A$ of $930.56 \mathrm{MeV}$. $\left(P^{+} / A\right.$ is the total energy per nucleon.) The distribution is not symmetric about its average value, as it would be if a simple Fermi gas model were used. Both of these effects are caused by the presence of nuclear mesons, which carry the remainder of the plus-momentum. 
The meson distributions are computed using

$$
f_{s}\left(k^{+}\right)=\int d^{2} k_{\perp}|\langle\Psi|a(\mathbf{k})| \Psi\rangle|^{2}
$$

for the scalar meson distribution, and

$$
f_{v}\left(k^{+}\right)=\int d^{2} k_{\perp} \sum_{\omega=1,3}|\langle\Psi|a(\mathbf{k}, \omega)| \Psi\rangle|^{2}
$$

for the vector meson distribution. The operator $a(\mathbf{k})$ destroys a scalar meson of momentum $k=\left(k^{+}, k_{\perp}\right)$, and $a(\mathbf{k}, \omega)$ destroys a vector meson of polarization $\omega$. The mean field approximation is used here, and the matrix elements needed to evaluate Eqs. (12) and (13) can be obtained using standard manipulations on Eqs. (5) and (6). The detailed expressions for $f_{s}, f_{v}$ have the same form as those of Ref. [15] in which the nucleus was treated as an elementary spherical source of scalar and vector mesons. The difference is that self-consistent field theory is used here to obtain the nucleon densities $\rho_{s, B}$. The scalar mesons are found to carry $4.70 \mathrm{MeV}$ of the plus-momentum, while the vector mesons carry $253.30 \mathrm{MeV}$ (or $27 \%$ ) of the nuclear plus-momentum. The technical reason for the difference is that the evaluation of $a^{\dagger}(\mathbf{k}, \omega) a(\mathbf{k}, \omega)$ counts vector mesons "in the air" and the resulting expression contains polarization vectors that give factor of $\frac{1}{k^{+}}$which enhance the distribution of vector mesons of low $k^{+}$. The results for the vector meson distribution are shown in Fig. 2.

It is worthwhile to see how the present results are related to lepton-nucleus deep inelastic scattering experiments. We find that the nucleons carry only $72 \%$ of the plus-momentum. The use of our $f_{N}$ in standard convolution formulae lead to a reduction in the nuclear structure function that is far too large ( $~ 95 \%$ is needed [1]) to account for the reduction observed [1] in the vicinity of $x \sim 0.5$. The reason for this is that the quantity $M+g_{s} \phi$ acts as a nucleon effective mass of about $670 \mathrm{MeV}$, which is very small. A similar difficulty occurs in the $\left(e, e^{\prime}\right)$ reaction [16] when the mean field theory is used for the initial and final states. The use of a small effective mass and a large vector potential enables a simple reproduction of the nuclear spin orbit force [8]. However, effects beyond the mean field may lead to a significant effective tensor coupling of the isoscalar vector meson [17 and to an increased 
value of the effective mass. Such effects are incorporated in Bruckner theory and a light front version [18] could be applied to finite nuclei with better success in reproducing the data.

In any case, these kinds of nuclear physics calculations can be done in a manner in which modern nuclear dynamics is respected, boost invariance in the $z$-direction is preserved, and in which the rotational invariance so necessary to understanding the basic features of nuclei is maintained. 


\section{REFERENCES}

[1] R.L. Jaffe, in Relativistic dynamics and quark-nuclear physics, edited by M.B. Johnson and A. Picklesimer (Wiley, New York, 1985); L.L. Frankfurt and M.I. Strikman, Phys. Rep. 160, 235 (1988); M. Arneodo, Phys. Rep. 240, 301 (1994); D.F. Geesaman, K. Saito, A.W. Thomas, Ann. Rev. Nucl. Part. Sci. 45, 337 (1995).

[2] G.B. West, Phys. Rep. 18C, 264 (1975).

[3] L. L. Frankfurt and M. I. Strikman Phys. Rep. 76, 215 (1981).

[4] X. Ji and B. W. Filippone, Phys. Rev. C42, R2279 (1990).

[5] R.P. Bickerstaff, M.C. Birse, and G.A. Miller, Phys. Rev. Lett.53, 2532 (1984); M. Ericson, and A.W. Thomas, Phys. Lett. 148B, 191 (1984); E.L. Berger, Nucl. Phys. B267, 231 (1986); D.M. Alde et al., Phys. Rev. Lett. 64, 2479 (1990).

[6] S. J. Brodsky, H-C Pauli, and S. S. Pinsky, Phys. Rep. 301, 299,(1998); M. Burkardt, Adv. Nucl. Phys. 23, 1 (1993); S. J. Brodsky and G. P. Lepage, in Perturbative quantum chromodynamics, edited by A. Mueller (World Scientific, Singapore 1989); X. D. Ji Comm. Nucl. Part. Phys. 21, 123 (1992); W.-M. Zhang, Chinese J. Phys. 32, 717 (1994); A. Harindranath, An Introduction to Light Front Dynamics for Pedestrians, hep-ph/9612244.

[7] P. A. M. Dirac, Rev. Mod. Phys. 21, 392 (1949).

[8] B.D. Serot and J.D. Walecka, Adv. Nucl. Phys. 16 (1986); Int. J. Mod. Phys. E6, 515 (1997).

[9] C.J. Horowitz and B.D. Serot, Nucl. Phys. A368, 503 (1981). We neglect here the electromagnetic effects as well as those of the $\rho$ meson.

[10] G.A. Miller, Phys. Rev. C56, R8 (1997); G.A. Miller, Phys. Rev. C56, 2789 (1997).

[11] D.E. Soper, Field theories in the infinite momentum frame, SLAC pub-137 (1971); D.E. 
Soper, Phys. Rev. D4, 1620 (1971); T-M Yan Phys. Rev. D7, 1760, 1780 (1974).

[12] A. Harindranath and W-M Zhang, Phys. Rev. D48, 4861, 4881, 4903 (1993).

[13] J.A. McNeil et al., Phys. Rev. C40, 399 (1989); W.R. Johnson et al., Phys. Rev.A 37, 307 (1988).

[14] Both sides of the Eqs. (5566) depend on $x=\left(x^{-}, \mathbf{x}_{\perp}\right)$, but changing variables using $z \equiv-x^{-} / 2, \boldsymbol{r} \equiv\left(\boldsymbol{x}_{\perp}, z\right)$, allows one to show that the mesonic equations take on a manifestly spherically symmetric form, with $\phi\left(x_{\perp}, x^{-}\right)=\phi\left(x_{\perp}^{2}+\left(x^{-} / 2\right)^{2}\right), V^{\mu}\left(x_{\perp}, x^{-}\right)=$ $\delta_{\mu, 0} V^{0}\left(x_{\perp}^{2}+\left(x^{-} / 2\right)^{2}\right)$.

[15] M. Burkardt and G.A. Miller, Phys. Rev. C58, 2450 (1998).

[16] H. Kim, C.J. Horowitz, and M.R. Frank, Phys. Rev. C51, 792 (1995).

[17] R.J. Furnstahl, J.J. Rusnak, and B.D. Serot Nucl.Phys. A632, 607 (1998).

[18] G.A. Miller and R. Machleidt, Light front theory of nuclear matter, preprint NT@UW98-21, submitted to Phys. Rev. Lett. 


\section{TABLES}

TABLE I. Comparison of the single particle spectra of ${ }^{40} \mathrm{Ca}$ in the equal time (ET) formalism $\left(\epsilon_{n}-M_{N}\right)$ with the light front $(\mathrm{LF}) \operatorname{method}\left(p_{n}^{-} / 2-M_{N}\right)$. Scalar and vector meson parameters are taken from Horowitz and Serot [9], and we have assumed isospin symmetry.

\begin{tabular}{|c|c|c|c|c|}
\hline \multicolumn{2}{|c|}{$\mathrm{ET}$} & \multicolumn{3}{|c|}{$\mathrm{LF}$} \\
\hline State $n$ & $\epsilon_{n}-M_{N}(\mathrm{MeV})$ & $J_{z}=1 / 2$ & $J_{z}=3 / 2$ & $J_{z}=5 / 2$ \\
\hline $0 \mathrm{~s}_{1 / 2}$ & -55.40 & -55.39 & & \\
\hline $0 \mathrm{p}_{3 / 2}$ & -38.90 & -38.90 & -38.90 & \\
\hline $0 \mathrm{p}_{1 / 2}$ & -33.18 & -33.18 & & \\
\hline $0 \mathrm{~d}_{5 / 2}$ & -22.75 & -22.75 & -22.75 & -22.74 \\
\hline $1 \mathrm{~s}_{1 / 2}$ & -14.39 & -14.36 & & \\
\hline $0 \mathrm{~d}_{3 / 2}$ & -13.87 & -13.87 & -13.88 & \\
\hline
\end{tabular}




\section{FIGURES}

FIG. 1. Nucleon plus-momentum distribution function, $f_{N}(y)$, for ${ }^{40}$ Ca. Here $y \equiv p^{+} /\left(M_{A} / A\right)$

FIG. 2. Vector meson plus-momentum distribution, $y f_{v}(y)$, for ${ }^{40} \mathrm{Ca}$. 


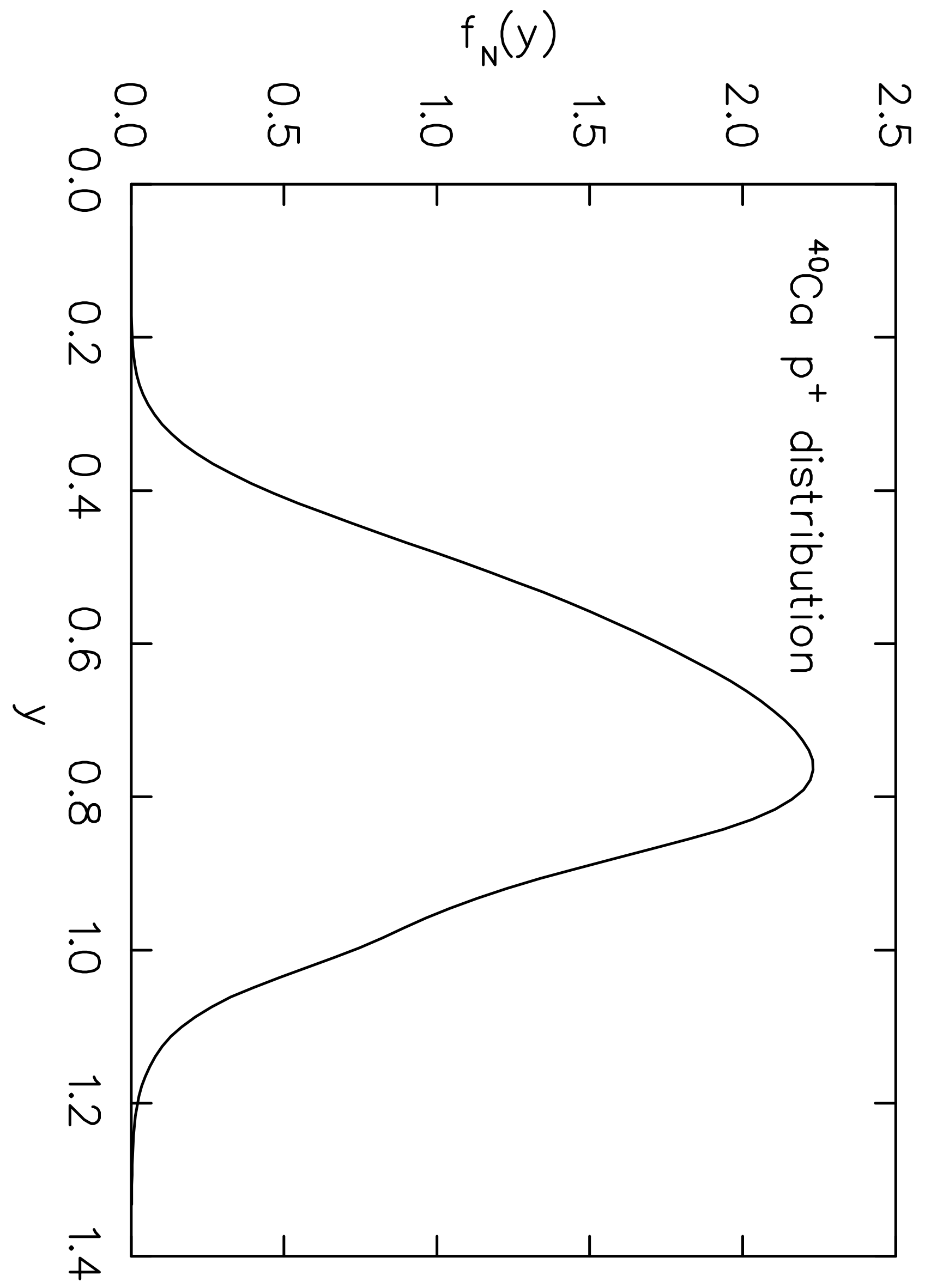




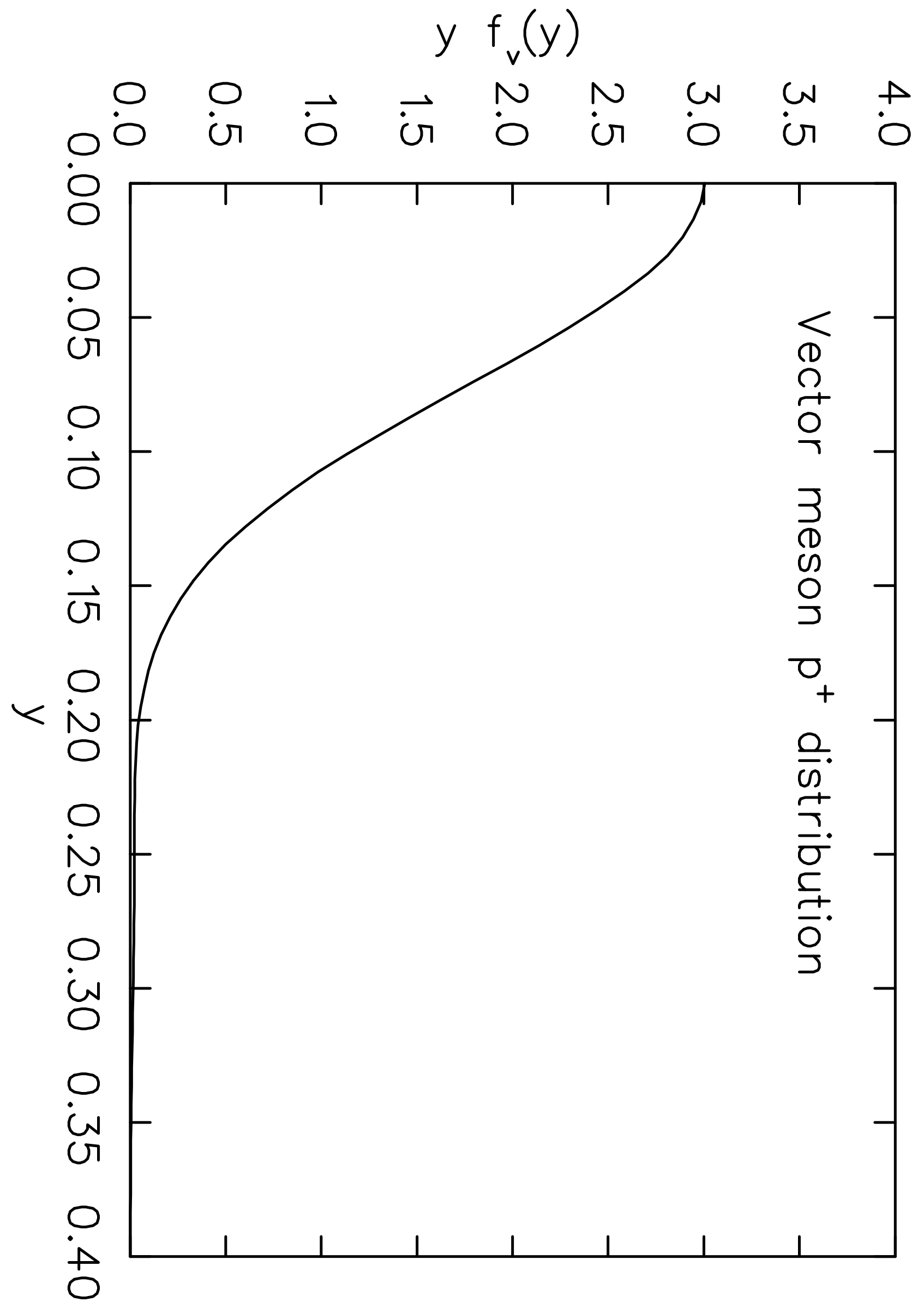

